\title{
Magnitude of Depression and Associated Factors among Mizan Aman Health Science Student Southern Ethiopia
}

Bereket Beyene Gebre ${ }^{1 *}$, Zebene Mekonnen² and Asres Bedaso ${ }^{1}$

${ }^{1}$ School of Nursing, College of Health Science, Hawassa University, Southern Ethiopia

${ }^{2}$ Department of Nursing, College of Health Science, Wolkite University, Southern Ethiopia

\begin{abstract}
Background: Depression is the most common mental disorder. It is a major cause of disability across the world. Depression can affect everyone and is not an exclusive disease.

Objective: This study was designed to assess magnitude and associated factors of depression among MizanAman College of health Science students from March 1-30, 2017.

Methods: An institution based cross sectional quantitative study was conducted on 328 college students and the subjects were identified by simple random sampling techniques after stratified by department with proportional sample allocation. Patient Health Questionnaires-9 (PHQ-9) is an instrument used to measure magnitude of depression and its associated factors. A pre-tested structured questionnaire was used and data were edited, coded and entered using Epi data version 3.1 and exported to SPSS version 21 for analysis. Then the independent variables were identified by forward binary logistic regression.

Results: The mean age of the respondents were 20.50 years $(S D=3.078)$. The prevalence of depression was $34.1 \%$. From those depressed; $88(26.83 \%), 22(6.71 \%)$ and $2(0.61 \%)$ had mild, moderate and severe depression respectively. According to this study, family history of depression, interest to learn in the department and presence of other disease (dyspepsia, malaria, headache were found to be significantly associated with depression among health science students.
\end{abstract}

Conclusion: The prevalence of depression in this study was relatively high. Therefore the college should provide counselling and advice for students on the independent predictors of depression.

Keywords: Magnitude; Depression; Patient Health Questionnaire (PHQ-9); College students

\section{Abbreviations}

CI: Confidence Interval; ETB: Ethiopian Birr; FDRE: Federal Democratic Republic of Ethiopia; MACOHS: Mizan Aman College of Health Science; NGOs: Non-Governmental Organizations; TLP: Teaching Learning Process; PHQ: Patient Health Questionnaire.

\section{Introduction}

\section{Background}

Depression is a common mental disorders with a wide range of mental health problems characterized by the absence of a positive affect (a loss of interest and enjoyment in ordinary things and experiences), low mood and a range of associated emotional, cognitive, physical and behavioral symptoms $[1,2]$. Depression is a very painful and difficult human experience [3]. It is a major cause of morbidity commonly associated with a decline in social, occupational and interpersonal functioning [4]. Depression is estimated to affect 340 million people globally [5]. It is the fourth most important contributor to the global burden of disease and comprises in year $2000,4.4 \%$ of the total disability adjusted life years [6,7].

Depression can affect everyone, is not an exclusive disease, and appears in both sexes and in all age groups and races, in addition to its hereditary aspects, is also caused by social and environmental factors [2]. It is projected to become the second most common cause by 2020 [8]. The mortality rate due to suicide is 20 times greater among depressed individuals than the general population [9-13].

It is estimated that by the year 2020 if current trends for demographic and epidemiological transition continues, the burden of depression will increase to $5.7 \%$ of the total burden of the disease and is expected to be the largest contributor to disease burden $[9,14]$.

\section{Objective}

General objective: To assess magnitude of depression and associated factors among Mizan-Aman college of health science students, Mizan-Aman, South West Ethiopia, 2017.

Specific objective: To determine prevalence of depression among college students at Mizan-Aman College of health science.

To identify association factors of depression among college students at Mizan-Aman College of health science.

\section{Materials and Methods}

\section{Study area and period}

This study was conducted in Mizan-Aman college of health science,

${ }^{*}$ Corresponding author: Bereket Beyene Gebre, School of Nursing, College of Health Science, Hawassa University, Southern Ethiopia, Tel: +25146220 5311; E-mail: bereket2007beyene@gmail.com

Received: April 12, 2019; Accepted: April 24, 2019; Published: April 30, 2019

Citation: Gebre BB, Mekonnen Z, Bedaso A (2019) Magnitude of Depression and Associated Factors among Mizan Aman Health Science Student Southern Ethiopia. J Psychiatry 22: 463. doi: 10.35248/2378-5756.19.22.463

Copyright: @ 2019 Gebre BB, et al. This is an open-access article distributed under the terms of the Creative Commons Attribution License, which permits unrestricted use, distribution, and reproduction in any medium, provided the original author and source are credited 
which is located in southern nation nationality people region (SNNPR), $832 \mathrm{~km}$ `s from Hawassa the capital city of the region. It is also $588 \mathrm{~km}$ 's from Addis Ababa, capital city of the country. The college has six departments which are comprehensive nursing, midwifery, health informatics, laboratory technology, emergency technician and health extension. It provides education for a total of 1442 students. The college has 90 academic staff and 59 supportive staffs. The study was conducted from March 2017-April 2017 G.C.

\section{Study design}

An institution based cross sectional study design was conducted.

\section{Source population}

Were all students who were registered in Mizan-Aman College of health science in 2016/17.

\section{Study population}

All sampled students who were registered in 2016/17 and found in the study period.

\section{Inclusion criteria}

Were students who attend at least one month on a class.

\section{Exclusion criteria}

Students who was sick to the extent of unable to read and write during data collection period and student who was absentee during date of data collection.

\section{Sample size determination}

The sample size is determined using a single population proportion formula as follows:

$n=\frac{(Z \alpha / 2)^{2}(1-p)}{D^{2}} n=\frac{(1.96)^{2} 5(1-1.5)}{(0.05)^{2}}=384$

$\mathrm{p}=$ Estimate of $\%$ prevalence of depression among college students;

D: Margin of sampling error tolerated-5\% (0.05);

$\alpha=$ Critical value at $95 \%$ confidence interval of certainty (1.96).

Since the source population is 1403 that is below 10,000 finite population correction is needed.

$$
n f=\frac{n}{1+n / N}=\frac{384}{1+384 / 1442}=303
$$

Where, $\mathrm{N}=$ total population $(1442)$ 336.

After adding non response rate of $10 \%$ the total sample size becomes

\section{Sampling technique}

Stratified random sampling method was used to classify students in a department with proportionate allocation. Then study subjects were selected by simple random sampling technique using list of names obtained from college registrar.

\section{Dependent variables}

Depression

\section{Independent variables}

Socio demographic characteristics: Age, Sex, Religion, Ethnicity,
Marital status, income, separation from family, family education and job status, family history of depression.

Teaching learning related factors: Interest to learn in the department, decrease grade than expected, missing many classes, problem in TLP.

Behavioral factors: Khat chewing, drinking alcohol, cigarette smoking, use of drugs for without prescription. Medical related factors: Asthma, heart diseases, hypertension, kidney diseases and others disease.

Medical related factors: Asthma, heart diseases, hypertension, kidney diseases and others disease.

\section{Data Collection Instruments and Method}

The data was collected by structured self-administered questionnaires. The questionnaire has four parts. The sociodemographic, teaching learning related, substance use and medical factors as predictor variables of depression The Patient Health Questionnaire (PHQ-9) was a self-administered measure designed for use in primary care and non-psychiatric settings. It contained items derived from the Diagnostic and Statistical Manual of Mental Disorders, $4^{\text {th }}$ Edition (DSM IV). Self-administered questionnaires' were given for selected students in their classrooms at the end of the class by the data collectors.

\section{Data analysis procedures}

After data collection, each questionnaire was checked visually for completeness. The responses was coded and entered into Epi data version 3.0 and exported to SPSS version 20 for analysis. Binary logistic regression will be carried out to assess the association of dependent variable with independent variables. Finally forward stepwise logistic regression model with all independent variables having $\mathrm{p}$ value $<0.25$ will be fitted and adjusted odds ratio will be calculated to identify independent predictors of depression among students.

\section{Data quality}

Properly designed data collection instrument was prepared in English and then translated to Amharic and back translated to English to check consistency. Data collectors will be oriented on the overall data collection procedure. Training will be given to data collectors and supervisors. Pre-test was made by five percent of the sample size in Aman Poly Technique College before the actual data collection to check the accuracy and validity of the questionnaire prior to the actual study period and the necessary adjustment was made accordingly.

\section{Operational definition}

Depression: According to this study the existence and prevalence of depression is determined by patient health questionnaire-9 (PHQ 9) scale and ranges from 0-4 is normal, 5-9 mild, 10-14 moderate, 15-19 severe and 20-27 very severe.

\section{Ethical Consideration}

Before the data collection, ethical clearance letter was obtained from ethical review committee of MACHOS. The letter was submitted to the departments for permission. The students were informed and their oral consent was obtained. 
Citation: Gebre BB, Mekonnen Z, Bedaso A (2019) Magnitude of Depression and Associated Factors among Mizan Aman Health Science Student Southern Ethiopia. J Psychiatry 22: 463. doi: 10.35248/2378-5756.19.22.463

Page 3 of 6

\section{Results}

Out of 336 respondents intended to be included in the study, 328 participants provided complete data and gave response rate of $97.6 \%$.

\section{Socio demographic characteristics}

In this study the majority of the respondents $236(72.0 \%)$ were female. The mean age and Standard Deviation (SD) of the participants was 20.50 years $(\mathrm{SD}=3.078)$. The age of participants between $17-23$ and $24-40$ were $284(86.6 \%)$ and $44(13.4 \%)$ respectively. Concerning marital status $234(71.3 \%)$ was single (Table 1$)$.

Teaching learning related factors: From the participants; 313 (95.4\%) students were responded that they didn't faced problems related to teaching learning process in Table 2.

Behavioural factors: From the total participants; 326 (99.4\%) and 316 (96.3\%) were never smoked cigarette and use drugs without prescription respectively (Table 3 ).

Medical illness related factors: From those responses of the participants; 326 (99.4\%) and 320 (97.6\%) hadn’t hypertension and asthma respectively shown in Table 4.

Magnitude and level of depression among college students: Magnitude of depression showing in Table 5.

\begin{tabular}{|c|c|c|}
\hline Variables & Frequency (n) & Percent (\%) \\
\hline \multicolumn{3}{|c|}{ Sex } \\
\hline Male & 92 & $28 \%$ \\
\hline Female & 236 & $72 \%$ \\
\hline \multicolumn{3}{|c|}{ Age in years } \\
\hline $17-23$ & 284 & $86.60 \%$ \\
\hline $24-40$ & 44 & $13.40 \%$ \\
\hline \multicolumn{3}{|c|}{ Religion } \\
\hline Orthodox & 151 & $46 \%$ \\
\hline Muslim & 43 & $13.1 \%$ \\
\hline Protestant & 129 & $39.3 \%$ \\
\hline Others & 5 & $1.5 \%$ \\
\hline \multicolumn{3}{|c|}{ Marital status } \\
\hline Single & 223 & $68.00 \%$ \\
\hline Married & 91 & $27.7 \%$ \\
\hline Divorced & 14 & $4.3 \%$ \\
\hline \multicolumn{3}{|c|}{ Ethnicity } \\
\hline kaffa & 92 & $28 \%$ \\
\hline Bench & 70 & $21.3 \%$ \\
\hline Shakka & 67 & $20.4 \%$ \\
\hline Amhara & 50 & $15.2 \%$ \\
\hline Oromo & 23 & $7 \%$ \\
\hline Wolayta & 18 & $5.5 \%$ \\
\hline Others & 8 & $2.4 \%$ \\
\hline \multicolumn{3}{|c|}{ Income status (ETB) } \\
\hline $0-299$ & 4 & $1.2 \%$ \\
\hline $300-499$ & 167 & $50.9 \%$ \\
\hline$\geq 500$ & 157 & $47.9 \%$ \\
\hline \multicolumn{3}{|c|}{ Departments } \\
\hline Clinical nurse & 82 & $25 \%$ \\
\hline Midwifery & 34 & $10.4 \%$ \\
\hline Health extension & 110 & $33.5 \%$ \\
\hline Laboratory technician & 52 & $15.9 \%$ \\
\hline Emergency technician & 9 & $2.7 \%$ \\
\hline
\end{tabular}

\begin{tabular}{|c|c|c|}
\hline Health informatics & 41 & $12.5 \%$ \\
\hline \multicolumn{3}{|c|}{ Father Educational status } \\
\hline Illiterate & 79 & $24.1 \%$ \\
\hline Read \& write only & 118 & $36 \%$ \\
\hline Primary & 33 & $10.1 \%$ \\
\hline Secondary & 39 & $11.9 \%$ \\
\hline College and above & 59 & $18 \%$ \\
\hline \multicolumn{3}{|c|}{ Mothers Education status } \\
\hline Illiterate & 118 & $36 \%$ \\
\hline Read \& write only & 112 & $34.1 \%$ \\
\hline Primary & 35 & $10.7 \%$ \\
\hline Secondary & 42 & 12.8 \\
\hline College and above & 21 & $6.4 \%$ \\
\hline \multicolumn{3}{|c|}{ Father job status } \\
\hline Yes & 205 & $62.5 \%$ \\
\hline No & 123 & $37.5 \%$ \\
\hline \multicolumn{3}{|c|}{ Mother job status } \\
\hline Yes & 152 & $46.3 \%$ \\
\hline No & 176 & $53.7 \%$ \\
\hline \multicolumn{3}{|c|}{ Family history of depression } \\
\hline Yes & 81 & $24.7 \%$ \\
\hline No & 247 & $75.3 \%$ \\
\hline
\end{tabular}

Table 1: Socio demographic characteristics of Mizan-Aman Health Science College students, Bench Maji zone, Southwest, Ethiopia, $2017(n=328)$.

\begin{tabular}{|c|c|c|}
\hline Teaching Learning related factors & Frequency $(n)$ & Percent (\%) \\
\hline \multicolumn{3}{|c|}{ Interest in the field of study } \\
\hline Yes & 269 & $82 \%$ \\
\hline No & 59 & $18 \%$ \\
\hline \multicolumn{3}{|c|}{ Decrease grade than expected } \\
\hline Yes & 136 & $41.5 \%$ \\
\hline No & 192 & $58.5 \%$ \\
\hline \multicolumn{3}{|c|}{ Missing many classes } \\
\hline Yes & 39 & $11.9 \%$ \\
\hline No & 289 & $88.1 \%$ \\
\hline \multicolumn{3}{|c|}{ Problem in TLP } \\
\hline Yes & 313 & $95.4 \%$ \\
\hline No & 15 & $4.6 \%$ \\
\hline
\end{tabular}

Table 2: Frequency distribution of teaching learning related factors among MizanAman Health Science College students, Bench Maji zone, Southwest, Ethiopia, $2017(n=328)$.

\begin{tabular}{|c|c|c|}
\hline Behavioral predictors & $\begin{array}{c}\text { Frequency (n) } \\
\text { Chat chewing }\end{array}$ \\
\hline \multicolumn{1}{|c|}{ Percent (\%) } \\
\hline Yes & 16 & $4.9 \%$ \\
\hline No & 312 & $95.1 \%$ \\
\hline & Alcohol drink \\
\hline Yes & 14 & $4.3 \%$ \\
\hline No & 314 & $95.7 \%$ \\
\hline & Cigarette smoke \\
\hline Yes & 2 & $0.6 \%$ \\
\hline No & 326 & $99.4 \%$ \\
\hline Yes & 12 & $3.7 \%$ \\
\hline No & 316 & $96.3 \%$ \\
\hline
\end{tabular}

Table 3: Frequency distribution of behavioral factors among Mizan-Aman Health Science College students, Bench Maji zone, Southwest, Ethiopia, 2017 ( $n=328$ ). 
Citation: Gebre BB, Mekonnen Z, Bedaso A (2019) Magnitude of Depression and Associated Factors among Mizan Aman Health Science Student Southern Ethiopia. J Psychiatry 22: 463. doi: 10.35248/2378-5756.19.22.463

Page 4 of 6

Level of depression is shown in Table 6.

Association of socio-demographic factors with depression: From 12 socio demographic variables, 5 variables like ethnicity, academic year, father education level, mother education level and family history of depression had significant association with depression in the binary logistic regression by $95 \% \mathrm{CI}, \mathrm{p}<0.25$ (Table 7 ).

Association of teaching learning factors with depression: Among the five teaching learning related factors; Interest to learn in the department and missing many class are significant with depression at $95 \% \mathrm{CI}$, and p-value $<0.25$ (Table 8 ).

Association of behavioral factors with depression: From behavioral factors only chewing of Khat had associated in binary logistic regression in Table 9.

Association of medical illness with depression: From those five medical illness related factors; Asthma, kidney disease, heart disease and having other diseases (malaria, headache, and dyspepsia) had

\begin{tabular}{|c|c|c|}
\hline Medical illness factors & Frequency (n) & Percent (\%) \\
\hline \multicolumn{3}{|c|}{ Asthma } \\
\hline Yes & 8 & $2.4 \%$ \\
\hline No & 320 & $97.6 \%$ \\
\hline \multicolumn{3}{|c|}{ Kidney disease } \\
\hline Yes & 46 & $14 \%$ \\
\hline No & 282 & $86 \%$ \\
\hline Yes & 15 \\
\hline No & 313 & $4.6 \%$ \\
\hline \multicolumn{3}{|c|}{ Heart disease } \\
\hline Yes & 2 \\
\hline No & 326 & $95.4 \%$ \\
\hline \multicolumn{2}{|c|}{ Hypertension } \\
\hline No you have others disease \\
\hline Dyspepsia & 275 & $9.6 \%$ \\
\hline Headache & 19 & $83.8 \%$ \\
\hline Malaria & 15 & $5.8 \%$ \\
\hline Others & 9 & $2.7 \%$ \\
\hline
\end{tabular}

Table 4: Frequency distribution of medical illness related factors among MizanAman Health Science College students, Bench Maji zone, Southwest, Ethiopia, $2017(n=328)$.

\begin{tabular}{|c|c|c|}
\hline Depression status & Frequency (n) & Percent (\%) \\
\hline No & 216 & $65.9 \%$ \\
\hline Yes & 112 & $34.1 \%$ \\
\hline Total & 328 & $100 \%$ \\
\hline
\end{tabular}

Table 5: Magnitude of depression among Mizan-Aman Health Science College students in Bench Maji zone, Southwest, Ethiopia, 2017 ( $n=328)$.

\begin{tabular}{|c|c|c|}
\hline Level of depression & Frequency (n) & Percent (\%) \\
\hline No depression (PHQ scale 0-4) & 216 & 65.85 \\
\hline Mild depression(PHQ scale 5-9) & 88 & 26.83 \\
\hline Moderate depression(PHQ scale 10-14) & 22 & 6.71 \\
\hline Severe depression(PHQ scale 15-19) & 2 & 0.61 \\
\hline Total & 328 & 100 \\
\hline
\end{tabular}

Table 6: Level of depression among Mizan-Aman Health Science College students, Bench Maji zone, Southwest, Ethiopia, $2017(n=328)$.

\begin{tabular}{|c|c|c|c|}
\hline $\begin{array}{l}\text { Socio- demographic } \\
\text { factors }\end{array}$ & \multicolumn{2}{|c|}{ Depression } & COR, $95 \% \mathrm{Cl}$ \\
\hline \multicolumn{4}{|c|}{ Sex } \\
\hline Male & $61(18.6 \%)$ & $31(9.5 \%)$ & 1 \\
\hline Female & $155(47.3 \%)$ & $81(24.7 \%)$ & $1.028(0.618-1.711)$ \\
\hline \multicolumn{4}{|c|}{ Age } \\
\hline $17-23$ & $186(56.7 \%)$ & $98(29.9 \%)$ & 1 \\
\hline $24-40$ & $30(9.1 \%)$ & $14(4.3 \%)$ & $0.886(0.449-1.748)$ \\
\hline \multicolumn{4}{|c|}{ Religion } \\
\hline Orthodox & $96(29.3 \%)$ & $55(16.8 \%)$ & 1 \\
\hline Muslim & $31(9.5 \%)$ & $12(3.7 \%)$ & $0.676(0.321,1.422)$ \\
\hline Protestant & $85(25.9 \%)$ & $44(13.4 \%)$ & $0.904(0.552,1.478)$ \\
\hline Others & $4(1.2 \%)$ & $1(0.3 \%)$ & $0.436(0.048,4.003)$ \\
\hline \multicolumn{4}{|c|}{ Marital status } \\
\hline Single & $143(43.6 \%)$ & $80(24.4 \%)$ & 1 \\
\hline Married & $61(18.6 \%)$ & $30(9.1 \%)$ & $0.879(0.525,1.472)$ \\
\hline Divorced & $12(3.7 \%)$ & $2(0.6 \%)$ & $0.298(0.065,1.365)$ \\
\hline \multicolumn{4}{|c|}{ Ethinicity } \\
\hline Kaffa & $55(16.8 \%)$ & $37(11.3 \%)$ & 1 \\
\hline Bench & $53(16.2 \%)$ & $17(5.2 \%)$ & $0.477(0.240,0.948)$ \\
\hline Shakka & $41(12.5 \%)$ & $26(7.9 \%)$ & $0.943(0.495,1.795)$ \\
\hline Amhara & $32(9.8 \%)$ & $18(5.5 \%)$ & $0.836(0.410,1.704)$ \\
\hline Oromo & $15(4.6 \%)$ & $8(2.4 \%)$ & $0.793(0.305,2.058)$ \\
\hline Wolayta & $14(4.3 \%)$ & $4(1.2 \%)$ & $0.425(0.130,1.392)$ \\
\hline Others & $6(1.8 \%)$ & $2(0.6 \%)$ & $0.495(0.095,2.590)$ \\
\hline \multicolumn{4}{|c|}{ Income (ETB) } \\
\hline $0-299$ & $3(0.9 \%)$ & $1(0.3 \%)$ & $0.673(0.068-6.630)$ \\
\hline $300-499$ & $108(32.9 \%)$ & $59(18.0 \%)$ & $1.103(0.697-1.747)$ \\
\hline$\geq 500$ & $105(32.0 \%)$ & $52(15.9 \%)$ & 1 \\
\hline \multicolumn{4}{|c|}{ Academic year } \\
\hline First year & $142(43.3 \%)$ & $60(18.3 \%)$ & 1 \\
\hline Second year & $51(15.5 \%)$ & $37(11.3 \%)$ & $1.717(1.021,2.888)$ \\
\hline Third year & $23(7.0 \%)$ & $15(4.6 \%)$ & $1.543(0.753,3.162)$ \\
\hline \multicolumn{4}{|c|}{ Father educational status } \\
\hline Illiterate & $52(15.9 \%)$ & $27(8.2 \%)$ & $1.093(0.534-2.239)$ \\
\hline Read and write & $85(25.9 \%)$ & $33(10.1 \%)$ & $0.817(0.415-1.61)$ \\
\hline Primary & $16(4.9 \%)$ & $17(5.2 \%)$ & $2.237(0.933-5.362)$ \\
\hline Secondary & $23(7.0 \%)$ & $16(4.9 \%)$ & $1.465(0.632-3.392)$ \\
\hline College and above & $40(12.2 \%)$ & $19(5.8 \%)$ & 1 \\
\hline \multicolumn{4}{|c|}{ Mother educational status } \\
\hline Illiterate & $74(22.6 \%)$ & $44(13.4 \%)$ & 1 \\
\hline Read and write & $72(22.0 \%)$ & $40(12.2 \%)$ & $0.934(0.546,1.599)$ \\
\hline Primary & $22(6.7 \%)$ & $13(4.0 \%)$ & $0.994(0.455,2.169)$ \\
\hline Secondary & $33(10.1 \%)$ & $9(2.7 \%)$ & $0.459(0.201,1.048)$ \\
\hline College and above & $15(4.6 \%)$ & $6(1.8 \%)$ & $0.673(0.243,1.861)$ \\
\hline \multicolumn{4}{|c|}{ Father job status } \\
\hline Yes & $132(40.2 \%)$ & $73(22.3 \%)$ & 1 \\
\hline No & $84(25.6 \%)$ & $39(11.9 \%)$ & $0.84(0.522-1.350)$ \\
\hline \multicolumn{4}{|c|}{ Mother job status } \\
\hline Yes & $102(31.1 \%)$ & $50(15.2 \%)$ & 1 \\
\hline No & $114(34.8 \%)$ & $62(18.9 \%)$ & $1.109(0.702-1.755)$ \\
\hline \multicolumn{4}{|c|}{ Family history of depression } \\
\hline Yes & $45(13.7 \%)$ & $36(11.0 \%)$ & $1.8(1.076-3.012)$ \\
\hline No & $171(52.1 \%)$ & $76(23.2 \%)$ & 1 \\
\hline
\end{tabular}

Table 7: Binary logistic regression model for socio-demographic variables among Mizan-Aman Health Science College students, Bench Maji zone, Southwest, Ethiopia, $2017(n=328)$. 
Citation: Gebre BB, Mekonnen Z, Bedaso A (2019) Magnitude of Depression and Associated Factors among Mizan Aman Health Science Student Southern Ethiopia. J Psychiatry 22: 463. doi: 10.35248/2378-5756.19.22.463

Page 5 of 6

\begin{tabular}{|c|c|c|c|}
\hline $\begin{array}{c}\text { Teaching learning } \\
\text { factors }\end{array}$ & \multicolumn{2}{|c|}{$\begin{array}{c}\text { Depression } \\
\text { No n (\%) }\end{array}$} & COR, 95\% $\mathbf{C l}$ \\
\hline \multicolumn{5}{|c|}{ Interest in the field of the study } \\
\hline Yes & $171(52.1 \%)$ & $98(29.9 \%)$ & 1 \\
\hline No & $45(13.7 \%)$ & $14(4.3 \%)$ & $0.543(0.284,1.039)$ \\
\hline \multicolumn{4}{|c|}{ Below grade than expect } \\
\hline Yes & $94(28.7 \%)$ & $42(12.8 \%)$ & $0.779(0.488,1.243)$ \\
\hline No & $122(37.2 \%)$ & $70(21.3 \%)$ & 1 \\
\hline \multicolumn{4}{|c|}{ Missing many class } \\
\hline Yes & $22(6.7 \%)$ & $17(5.2 \%)$ & $1.578(0.8,3.111)$ \\
\hline No & $194(59.1 \%)$ & $95(29.0 \%)$ & 1 \\
\hline \multicolumn{5}{|c|}{ Satisfy by TLP } \\
\hline Yes & $205(62.5 \%)$ & $108(32.9 \%)$ & 1 \\
\hline No & $11(3.4 \%)$ & $4(1.2 \%)$ & $0.69(0.215,2.219)$ \\
\hline
\end{tabular}

Table 8: Binary logistic regression model for teaching learning factors among Mizan-Aman Health Science College students, Bench Maji zone, Southwest, Ethiopia, $2017(n=328)$.

\begin{tabular}{|c|c|c|c|}
\hline $\begin{array}{c}\text { Behavioral } \\
\text { factors }\end{array}$ & \multicolumn{2}{|c|}{$\begin{array}{c}\text { Depression } \\
\text { No n (\%) }\end{array}$ Yes n (\%) } & COR, 95\% CI \\
\hline \multicolumn{4}{|c|}{ Khat chewing } \\
\hline Yes & $8(2.4 \%)$ & $8(2.4 \%)$ & $2(0.730-5.479)$ \\
\hline No & $208(63.4 \%)$ & $104(31.7 \%)$ & 1 \\
\hline \multicolumn{4}{|c|}{ Alcohol intake } \\
\hline Yes & $9(2.7 \%)$ & $5(1.5 \%)$ & $1.075(0.351,3.282)$ \\
\hline No & $207(63.1 \%)$ & $107(32.6 \%)$ & 1 \\
\hline \multicolumn{4}{|c|}{ Using drugs without prescription } \\
\hline Yes & $8(2.4 \%)$ & $4(1.2 \%)$ & $0.963(0.284-3.27)$ \\
\hline No & $208(63.4 \%)$ & $108(32.9 \%)$ & 1 \\
\hline
\end{tabular}

Table 9: Binary logistic regression for behavioral factors among Mizan-Aman Health Science College students, Bench Maji zone, Southwest, Ethiopia, 2017 $(n=328)$.

\begin{tabular}{|c|c|c|c|}
\hline $\begin{array}{l}\text { Medical illness } \\
\text { related factors }\end{array}$ & $\begin{array}{l}\text { Depre } \\
\text { No } n(\%)\end{array}$ & $\begin{array}{l}\text { sion } \\
\text { Yes n (\%) }\end{array}$ & COR, $95 \% \mathrm{Cl}$ \\
\hline \multicolumn{4}{|c|}{ Asthma } \\
\hline Yes & $3(0.9 \%)$ & $5(1.5 \%)$ & $3.318(0.778-14.1457)$ \\
\hline No & $213(64.9 \%)$ & $107(32.6 \%)$ & 1 \\
\hline \multicolumn{4}{|c|}{ Kidney disease } \\
\hline Yes & $6(1.8 \%)$ & $9(2.7 \%)$ & $1.956(1.041-3.673)$ \\
\hline No & $210(64.0 \%)$ & $103(31.4 \%)$ & 1 \\
\hline \multicolumn{4}{|c|}{ Heart disease } \\
\hline Yes & $210(67.1 \%)$ & $6(40 \%)$ & $3.058(1.06-8.823)$ \\
\hline No & $103(32.9 \%)$ & $9(60 \%)$ & 1 \\
\hline \multicolumn{4}{|c|}{ Hypertension } \\
\hline Yes & $1(0.3 \%)$ & $1(0.3 \%)$ & $1.937(0.12-31.2610$ \\
\hline No & $215(65.5 \%)$ & $111(33.8 \%)$ & 1 \\
\hline \multicolumn{4}{|c|}{ Having others diseases other than the above } \\
\hline No & $189(57.6 \%)$ & $86(26.2 \%)$ & 1 \\
\hline Yes & $27(8.2 \%)$ & $26(7.9 \%)$ & $2.116(1.166,3.840)$ \\
\hline
\end{tabular}

Table 10: Binary logistic regression for medical illness factors among Mizan-Aman Health Science College students, Bench Maji zone, Southwest, Ethiopia, 2017 $(n=328)$.

significant association in binary logistic regression by CI: $95 \%, \mathrm{p}<0.25$ in (Tables 10 and 11).

\section{Discussion}

According to this study, the overall prevalence of depression was $34.1 \%$. From depressed participants; $26.83 \%$ had mild depression,

\begin{tabular}{|c|c|c|c|}
\hline Independent predictors & COR, 95\%CI & AOR, 95\%Cl & p \\
\hline Department choice based on interest of the students \\
\hline Yes & 1.00 & 1.00 & \\
\hline No & $0.543(0.284,1.039)$ & $0.51(0.239-0.947)$ & 0.034 \\
\hline \multicolumn{4}{|c|}{ Family history of depression } \\
\hline Yes & $1.8(1.076-3.012)$ & $1.964(1.142-3.378)$ & 0.029 \\
\hline No & 1.00 & 1.00 & \\
\hline & Others diseases & \\
\hline No & 1.00 & 1.00 & 0.032 \\
\hline Yes & $2.116(1.166,3.840)$ & $1.945(1.058,3.574)$ & 0 \\
\hline
\end{tabular}

Table 11: Independent predictors of depression among Mizan-Aman College of Health Science students, Bench Maji zone, Southwest, Ethiopia, 2017 ( $n=328)$.

$6.71 \%$ had moderate depression, $0.61 \%$ had severe depression but there was no very severe depression in this study. Similarly the overall prevalence of depression was similar with study done in student with low socio economic status in which $31.2 \%$ were depressed. This study were also similar with study done in Addis Ababa university among 1st year students and Ambo university students in which $27.7 \%$ and $32.2 \%$ were depressed respectively [15].

In this study, family history of depression had significant association with depression and similar with the study done in India on medical students [16].

This study also showed that the interest to learn in the department had a significant association with depression with study done in Iran interest in field of study, $(\mathrm{p}<0.001)$ [2].

And also according to this study; students who had medical illness like headache, dyspepsia, malaria, anaemia and typhoid fever had significant association with depression. This is a scientific truth that when individuals are diseased, the patients are more prone to be depressed due to the diseased process occurring on them.

\section{Limitation of the Study}

The study design was cross sectional nature of the study so it is snap shot and could not confirm cause and effect relationship.

\section{Conclusion}

The prevalence of depression among college students was relatively high. Family history of depression, interest to learn the department and students who had disease was the independent predictors of depression.

\section{Recommendation}

The Mizan-Aman health Science College should also communicate with the regional health office and concerned body during department selection or choice by students to be based on their interest. The college should also provide health education based on associated factors that might bring depression on student.

\section{Declaration}

\section{Ethics approval and consent to participate}

The study was conducted after approval of the proposal by Ethical Review Committee of MACOHS. Written informed consent was obtained from each study participant by assuring privacy and confidentiality throughout the data collection period in the college. 
Citation: Gebre BB, Mekonnen Z, Bedaso A (2019) Magnitude of Depression and Associated Factors among Mizan Aman Health Science Student Southern Ethiopia. J Psychiatry 22: 463. doi: 10.35248/2378-5756.19.22.463

There was no risk or hazardous procedures putting the participants at harm.

\section{Consent to publish}

The consent to publish was reached with my authors.

\section{Availability of data and materials}

The data supporting and the finding will be attached to editorial office if necessarily since it is avail in the corresponding author hand.

\section{Competing interests}

There is no competing interest.

\section{Funding}

No funding is required.

\section{Author's contribution}

The authors contribute for this study is conducting and preparing this manuscript.

\section{Acknowledgement}

First of all we would like to acknowledge Mizan Aman Health Science College in supporting to conduct this study.

\section{References}

1. Feyera F, Mihretie G, Bedaso A, Gedle D, Kumera G (2015) Prevalence of depression and associated factors among Somali refugee at melkadida camp, southeast Ethiopia: a cross-sectional study. BMC Psychiatry 15:171.

2. Leicester (2010) Depression in Adults with a Chronic Physical Health Problem Treatment and Management NICE Clinical Guidelines. National Collaborating Centre for Mental Health, British Psychological Society, United Kingdom.

3. https://www.covwarkpt.nhs.uk/iapt

4. Kaur S, Deepti SS, Lal M (2014) Prevalence and Correlates of Depression among College going students of District Amritsar, India. Int Res J Med Sci 2 : $5-9$

5. Berardi D, Leggieri G, Ceroni GB, Rucci P, Pezzoli A, et al. (2002) Depression in primary care nationwide epidemiology survey. Fam Pract 19: 397-400.

6. Kastrup CM, Ramos BA (2007) Global mental health. Dan Med Bull 54: 42-43.

7. Integrating mental health into primary care-A global perspective. WHO Geneva, Switzerland.

8. Safiri S, Khanjani N, Kusha A, Narimani MR, Karamzad N (2013) Prevalence of depression and its associated factors using Beck Depression Inventory among students of School of Health and Nutrition, Tabriz, Iran, in 2009. J Analyt Res Clin Med 1: 83-89.

9. Devi K, Patel R, Phil MA (2013) Study of Psychological Depression and its associated factors among Medical Students in Pondicherry. Indian J Basic and Applied Med Res 2: 1009-1016.

10. Depression: A Global Crisis World Mental Health Day. WHO (2012).

11. Lépine JP, Briley M (2011) The increasing burden of depression. Neuropsychiatr Dis Treat 7: 3-7.

12. Ferrari AJ, Charlson FJ, Norman RE, Patten SB, Freedman G, et al. (2013) Burden of depressive disorders by country, sex, age, and year: findings from the global burden of disease study 2010. PLoS Med 10: e1001547.

13. Birhanu A, Hassein K (2016) Prevalence and Factors Associated to Depression Among Ambo University Students, Ambo, West Ethiopia. J Health Med Nurs 25: 1-9.

14. Kumar GS, Jain A, Hegde S (2012) Prevalence of depression and its associated factors using Beck Depression Inventory among students of a medical college in Karnataka. Indian J Psychiatry 54: 223-226.

15. Tomoda A, Mori K, Kimura M, Takahashi T, Kitamura T (2000) One-year prevalence and incidence of depression among first-year university students in Japan: a preliminary study. Psychiatry Clin Neurosci 54: 583-588.

16. Berhanu Y (2015) Prevalence of Depression and Associated Factors among Addis Ababa University Students, Addis Abeba, Ethiopia. J Multidiscip Res Healthcare 2: 73-90 